\title{
Principles of fatigue in residency education: a qualitative study
}

\author{
Taryn S. Taylor MD, Christopher J. Watling MD PhD, Pim W. Teunissen MD PhD, \\ Tim Dornan MD PhD, Lorelei Lingard PhD
}

\section{Abstract}

Background: Proposals to implement fatigue-management strategies in residency education assume that medicine shares the view of other risk-adverse industries that fatigue is hazardous. This view is an essential underpinning of fatigue-management strategies that other industries have embedded as part of their workplace occupational health and safety programs. We sought to explore how residents understand fatigue in the context of their training environment.

Methods: We interviewed 21 residents in 7 surgical and nonsurgical programs at Western University in 2014. All participants met the inclusion criteria of routinely working 24-hour call shifts while enrolled in their training program. Data collection and analysis occurred iteratively in keeping with constructivist grounded theory methodology and informed theoretical sampling to sufficiency.

Results: Four predominant principles of fatigue captured how the social learning environment shaped residents' perceptions of fatigue. These included the conceptualization of fatigue as (a) inescapable and therefore accepted, (b) manageable through experience, (c) necessary for future practice and (d) surmountable when required.

Interpretation: This study elaborates our understanding of how principles of fatigue are constructed and reinforced by the training environment. Whereas fatigue is seen as a collective hazard in other industries, our data showed that, in residency training, fatigue may be seen as a personal challenge. Consequently, fatigue-management strategies that conceptualize fatigue as an occupational threat may have a limited impact on resident behaviour and patient safety.

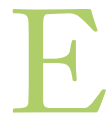

merging research has suggested that restrictions on resident duty hours may have done more harm than good. ${ }^{1-4}$ In Canada, the National Steering Committee on Resident Duty Hours proposed fatigue-management strategies as a promising alternative to prescriptive "one-size-fitsall" restrictions on resident duty hours. ${ }^{5,6}$ The final report advocated for new accreditation standards that would require residency programs to "develop, and keep up to date, fatigue risk management plans." ${ }^{~}, 6$ Empirical evidence supports the notion that prolonged sleep deprivation leads to performance impairment, ${ }^{7-10}$ and fatigue-management strategies would fulfill a need to maintain public accountability in the absence of duty-hour legislation. However, as Pattani and colleagues ${ }^{6}$ note, this mandate lacks important details about the design and implementation of fatigue-management strategies.

Unlike the explicit policies that limit resident duty hours in other countries, there is no model for fatigue-management strategies in medical education, and research about effectively managing fatigue in residency contexts is scarce. Arora and colleagues ${ }^{11}$ found that an educational seminar to improve sleep-hygiene practices had minimal impact on residents' behaviour. Policies of protected sleep time during prolonged duty periods have shown inconsistent results. ${ }^{12,13}$ Additional studies have highlighted how the influence of local training culture can trump duty-hour policy ${ }^{14}$ and alter perceptions of the impact of sleep deprivation. ${ }^{15}$ These varied perceptions and cultural influences have received little attention to date amid discussions of anticipated fatigue-management strategies.

In other industries, such as transportation, aviation and the military, fatigue-management strategies benefit from a uniform understanding of fatigue as an occupational threat. ${ }^{16}$ The fact that residents and faculty hold varied perceptions about the impact of fatigue on clinical performance, particularly within surgical contexts, suggests that fatigue may not be quite so straightforwardly understood in residency training. ${ }^{15,17}$ Until we know more about how fatigue is understood in residency training across multiple disciplines, we risk developing fatigue-management strategies that are irrelevant and

Competing interests: None declared.

This article has been peer reviewed.

Correspondence to: Taryn Taylor, ttaylor2010@meds.uwo.ca CMAJ Open 2016. DOI:10.9778/cmajo.20150086 
fail to address the existing shortcomings of duty-hour restrictions. Thus, we sought to explore how residents understand fatigue in the context of their training environment.

\section{Methods}

We used a constructivist grounded theory approach, which is suitable for socially situated research questions. ${ }^{18}$

\section{Participants and setting}

We conducted 21 individual, semistructured interviews at 3 hospitals within a single academic training centre at Western University with radiology, psychiatry, critical care, general surgery, pediatrics, internal medicine and orthopedic surgery residents. We deliberately sampled across these programs because the nature of residents' on-call work varied, and we wanted to include programs that have had limited representation in the existing duty-hours literature. All participants were routinely scheduled to work 24 -hour call shifts, as per our inclusion criteria.

\section{Recruitment}

Recruitment, data collection and analysis took place between December 2013 and June 2014. Recruitment involved email and face-to-face invitations approved by each program director. In keeping with the constructivist grounded theory method, we progressed from convenience to purposive and then theoretical sampling to sufficiency. ${ }^{19}$

\section{Data collection}

One co-investigator (T.T., a resident at one of the hospitals) conducted 20 of the 21 interviews in person or by telephone at the participant's convenience, often at the hospital. A co-investigator (L.L., a nonclinician scientist) deliberately conducted 1 interview for the purposes of rigour, to ensure that T.T.'s identity as a resident did not have a substantial impact on the information that participants chose to disclose during the open-ended interviews..$^{20}$ To remain reflexively aware of how her insider experience informed the analysis, T.T. made regular memos and coanalyzed with noninsider members of the research team. ${ }^{18,21}$

\section{Analysis}

Analyzed data included transcripts of the audiorecorded interviews, alongside field notes and analytical memos written by T.T. We followed the iterative process of data collection and analysis, which is characteristic of constructivist grounded theory. ${ }^{18}$ This required revisions of the semistructured interview guide (Appendix 1, available at www.cmajopen. $\mathrm{ca} /$ content/4/2/E200/suppl/DC1) in response to progressive refinement of the emerging theory. We chose not to confine the interviews to any specific definition of fatigue (e.g., physiologic, cognitive, emotional, chronic, acute) to allow for a more inclusive representation of fatigue.

We began our analysis with initial grounded theory coding with gerunds, followed by constant comparative analysis, which led to a more conceptual coding framework. ${ }^{18}$ Further refinement of this framework through collaborative analysis by all of us resulted in the final emergent theory. We used NVivo qualitative analysis software and MindNode Pro, a mind-mapping tool, to organize the coding process.

\section{Ethics approval}

We obtained research ethics approval from the institutional research ethics board at Western University (\#102769). Informed consent was obtained in writing from each participant.

\section{Results}

Twenty-one participants were sampled from a range of postgraduate years (1-7) and included 13 male residents, 12 married residents, 6 residents with children and 13 senior-level residents, as defined by the individual programs.

Residents referred to various features of their training environment that informed how they recognized, discussed and responded to fatigue in the context of residency training. Residents reported that "recognizing that you are tired" was always in tension with "also recognizing that there is a job to be done" (resident no. 018). In fact, some perceived that a goal of wellness was incompatible with the training they had chosen: "I am in this not to ... how do I put it correctly? Not for wellness ... I would have picked a different career. I'm in here to become a good doctor. The best way I can do that is to spend the hours seeing the things that need to be seen" (005). Such reflections suggest that understandings of fatigue were socially informed by tacit messaging and normative expectations about what residency is about.

Four "principles of fatigue" captured these socially informed ways in which residents made sense of fatigue. Fatigue was seen as (a) inescapable and therefore accepted, (b) manageable through experience, (c) necessary for future practice and (d) surmountable when required. Each principle is described below and illustrated with salient quotations from the interviews, which are provided in Table 1.

According to the principle of fatigue being inescapable and therefore accepted, fatigue is so pervasive that it is simply part of the job. Senior residents were predominant among those who endorsed the notion that fatigue is manageable through experience; they suggested that working while sleep deprived was a skill to be honed with practice. The principle that fatigue is necessary for future practice meant that working while fatigued was a valuable rite of passage to prepare for similar realities after residency. Residents across all sampled programs reinforced the idea that fatigue is surmountable when required, by suggesting that they could willfully persevere and continue to function in spite of fatigue.

The principle that fatigue is surmountable when required warrants further elaboration because it was prominent in participants' responses and is relevant to the concept of fatigue management. For many residents, fatigue was simply another challenge to overcome: "I think as long as you can stand up and stay awake and physically manage, then you can usually force your brain to work, even if it is harder to do and you may be more prone to mistakes" (017). Surmounting fatigue was not always presented as onerous. At times, participants 
implied that it was a natural response to workplace demands: "Usually when the volume is high and you know you have that pressure, it's almost like there's so much adrenaline, that I don't really feel as fatigued at that point" (010).

Although most residents agreed that fatigue would not stand in the way of doing what needed to be done, the principle of surmounting fatigue was not necessarily synonymous with providing high-quality care. One resident reflected on this by saying, "Obviously, we say we think we are doing a good job. But I think, maybe, you are just a little bit slower, less efficient. You could get things done faster and maybe more safely if people weren't as tired" (015). Various strategies within the workplace environment allowed participants to "push through" (018) in spite of fatigue. They described "filtering more to critical versus noncritical issues" (005), relying on "little checkboxes" to remember details (017), delegating tasks among team members to "distribute the workload" when feasible (014), and going "into autopilot" mode (010). Residents frequently spoke about how fatigue affected their mood and interactions with others: "I think we can all agree that the more fatigued we are, the more likely we are to have our social skills compromised" (010). This resident explained how the impact of fatigue on communication was perceived as advantageous in some circumstances: "I'm probably less articulate on a postcall breaking bad news, but at the same time, I'll just slow down and it may seem like I'm more empathetic ... which is really just because I'm having a hard time coming up with the words" (007).

In keeping with the principle that fatigue is surmountable when required, few residents directly attributed near misses or medical errors to fatigue. Residents' comments suggested that they typically needed to have negative outcomes drawn to their attention before they would attribute them to fatigue: "Until it was obvious that I was making technical mistakes, I probably wouldn't recognize it" (013). It seemed that fatigue was not a legitimate reason for making mistakes because it was deemed surmountable: "You know, being tired might be a valid excuse to put things off that can wait, but it's not an acceptable excuse for poor decision-making. At least not in the medical culture, I think" (006).

Some residents pointed to systems-based limitations that implied that surmounting fatigue was something they felt "forced to do" (008). For instance, one resident commented on the lack of "resources in place for staff physicians to be able to say, 'Hey, I am tired today' ... our groups aren't built that way ... your colleagues will have to pick up the slack and you can't call in a substitute like you might in another profession" (003). Thus, residents' workplace learning environments reinforced the idea that it is both necessary and plausible to "tough it out" (003) and overcome the effects of fatigue.

There were discrepancies across these 4 principles, where residents either challenged the prevailing principle or offered a variation. One questioned how the pervasiveness of fatigue has remained acceptable in medical training: "But at what point do we become where that is okay? Because in other professions, it is not okay, so, pilots or truck drivers" (018). A few residents disputed the idea that fatigue served a training purpose: "I don't believe we need to train people to perform well when we're tired" (013). There were a few, typically extreme, scenarios in which residents described insurmountable fatigue. One resident recalled a time when his attending physician, as he put it, "pulled the parachute and left" (020):

[My staff] operated all day and then he was on call with me all night and operated, and then the next day he operated all day ... I think it was 42 hours straight in the operating room. ... He called another staff to help finish the [last] case for him because he couldn't go on anymore. He was pooched (020).

Table 1: Four principles of fatigue

Principle

Quotation (anonymous resident no.) and therefore accepted

Fatigue is manageable through experience

Fatigue is necessary for future practice
Fatigue is inescapable

I think it's kind of expected. Everyone knows you're going to be tired. (012)

I don't think, culturally, talking about being tired is really appropriate in my program. We accept it. (013)

You just can't stand up anymore. You can't keep your eyes open anymore. You feel like you are going to pass out or whatever. A lot of people have those sensations after staying up for 24 hours. We learn to manage that. (017)

I'm sure people will disagree, and this seems silly, but being able to tolerate sleep deprivation and being able to do high-level performance while tired is trainable like fitness or something, I think. (019)

The program and consultants will make that statement, saying, we need to have a residency program where our residents are tired, because they need to be trained for the situation when they're in their community or they're in an environment where they don't have the luxury of sleep. (013)

In times of crisis, you need to still be able to make decisions and not freeze and be like, "Oh, I'm tired. I have to go to bed." I think that is what part of this training is ... we all learn to manage this in some way, I think. We all learn. (005)

Fatigue is surmountable when required
I think recognizing that you are tired but then, also recognizing that there is a job to be done. You are looking after patients. And so someone is there for your services. ... So, you just get to a point where ... you are just able to put your own needs secondary. "Hey, I'm tired. I'm not thinking quite the same and all of those things. But I have a job to do here." (018)

Recently, as a senior resident you don't have as many nights where you are up all night, but you have way more longer stretches where you are going 3 or 4 hours a night every night for 7 or 8 days. And you are amazed at the levels you can push yourself. (019) 
By pushing the limits of these socially accepted principles of fatigue, residents were reinforcing the existence of these principles while questioning their merit in the training environment.

\section{Interpretation}

Our study describes 4 principles of fatigue that residents use to make sense of fatigue within the training environment: fatigue is understood as inescapable, manageable, necessary and surmountable. These principles are more than individual beliefs; they seem to be deeply ingrained in the local training culture. And they reveal 3 serious problems, which must be faced before we can ensure the successful design and implementation of fatigue-management strategies in residency education.

The first problem is that these principles of fatigue have been discredited as myths in other high-performance industries, such as aviation and transportation. ${ }^{22}$ There are both regulatory and organizational reasons for why these industries have outgrown the principles of fatigue that residents in our study subscribe to. At a regulatory level, fatigue was first legitimized as a safety priority during a Department of Transportation/Federal Highway Administration Safety Summit 20 years ago. ${ }^{22}$ Since then, high-profile disasters, such as "the Chernobyl nuclear reactor meltdown, the Exxon Valdez catastrophe [and] the Three Mile Island nuclear power station accident," have clearly implicated fatigue as a "causal or contributory factor." ${ }^{23}$ Therefore, organizations that fail to implement sufficient fatigue-management systems are liable to be found "criminally negligent in the event of an on-the-job mishap." 2 Public visibility may be one distinguishing feature that has prompted other industries to adopt the stance that fatigue is an occupational safety threat, which, in turn, supports an explicit and formalized approach to fatigue management. By contrast, the consequences of fatigued residents are generally less public, with fewer casualties per fatigue-related incident, enabling continued skepticism in medical education about whether fatigue is dangerous in residency training. ${ }^{24}$

The second problem is that the principles revealed in our study characterize fatigue not as a threat, but as a challenge. Previous research has shown that surgical residents believe themselves to be resilient to the effects of sleep deprivation ${ }^{15}$ and that general surgery residents and faculty believe fatigue is irrelevant to patient care. ${ }^{17}$ Our research suggests that this belief may permeate residency more broadly. Even residents who perceived that their performance was negatively affected by fatigue nevertheless believed there had been no meaningful impact on patient care. In addition to not seeing fatigue as a threat, our participants perceived it as a challenge: the ability to keep working while fatigued was presented as a necessary skill. One implication of this is that the responsibility for this skill lies primarily with the resident. This is in stark contrast to other industries, such as transportation and aviation, ${ }^{25}$ that approach fatigue as a collective threat, not as a personal challenge.

The third problem is that residents cannot be expected to view fatigue as a threat when the working environment presents no acceptable alternative to working fatigued. Residents noted that there weren't any explicit provisions for addressing fatigue in the workplace, although there were established conventions for dealing with overwhelming patient volume, clinical uncertainty or procedural supervision. Studies of residency as a workplace training environment have shown that the workplace can implicitly preserve the status quo. ${ }^{26}$ As our data demonstrated, the unspoken message from the training environment was that fatigue must be surmountable, because it could not be helped.

\section{Limitations}

We conducted this research using a constructivist grounded theory method. This method is not intended to create a generalizable theory but, rather, to offer in-depth insight into a social phenomenon within a given context. We performed this research at a single centre, and the local training culture necessarily informs our results. Further research that explores and elaborates on our emergent theory in other contexts is needed. We relied on individual residents' accounts of experiencing fatigue rather than an objective measurement of fatigue because our research question was focused on residents' understanding. Consequently, this study does not quantify the prevalence of fatigue or attempt to measure its impact on patient care. We benefitted from having an insider (T.T.) who could provide analytical insights that were accessible to someone with lived experience ${ }^{21}$ as part of the research team. T.T. followed standard techniques for attending to her insider role throughout the analytical process. ${ }^{18,21}$

\section{Conclusion}

The success of fatigue-management strategies in other industries is based on the assumption that fatigue is uniformly understood as an occupational threat. Our results suggest that this assumption does not hold in residency. Under present conditions, the implementation of fatigue-management strategies in residency training may not have a substantial impact on resident fatigue or patient safety. Future research that explores whether existing principles of fatigue can be altered by changes within the training environment could bring us closer to successfully implementing effective fatigue-management strategies.

\section{References}

1. Carpenter RO, Spooner J, Arbogast PG, et al. Work hours restrictions as an ethical dilemma for residents: a descriptive survey of violation types and frequency. Curr Surg 2006;63:448-55.

2. Sen S, Kranzler HR, Didwania AK, et al. Effects of the 2011 duty hour reforms on interns and their patients: a prospective longitudinal cohort study. FAMA Intern Med 2013;173:657-62.

3. Desai SV, Feldman L, Brown L, et al. Effect of the 2011 vs 2003 duty hour regulation-compliant models on sleep duration, trainee education, and continuity of patient care among internal medicine house staff: a randomized trial. $7 A M A$ Intern Med 2013;173:649-55.

4. Parshuram CS, Amaral AC, Ferguson ND, et al. Patient safety, resident wellbeing and continuity of care with different resident duty schedules in the intensive care unit: a randomized trial. CMA7 2015;187:321-9.

5. National Steering Committee on Resident Duty Hours. Fatigue, risk and excellence: towards a Pan-Canadian consensus on resident duty hours. Ottawa: Royal College of Physicians and Surgeons of Canada; 2013: 1-52.

6. Pattani R, Wu PE, Dhalla IA. Resident duty hours in Canada: past, present and future. CMA7 2014;186:761-5.

7. Arnedt JT, Owens J, Crouch M, et al. Neurobehavioural performance of residents after heavy night call vs after alcohol ingestion. 7AMA 2005;294: $1025-33$.

8. Ayas NT, Barger LK, Cade BE, et al. Extended work duration and the risk of self-reported percutaneous injuries in interns. FAMA 2006;296:1055-62. 
9. Van Dongen HPA, Maislin G, Mullington JM, et al. The cumulative cost of additional wakefulness: dose-response effects on neurobehavioral functions and sleep physiology from chronic sleep restriction and total sleep deprivation. Sleep 2003;26:117-26.

10. Sugden C, Housden CR, Aggarwal R, et al. Effect of pharmacological enhancement on the cognitive and clinical psychomotor performance of sleepdeprived doctors: a randomized controlled trial. Ann Surg 2012;25 5:222-7.

11. Arora VM, Georgitis E, Woodruff JN, et al. Improving sleep hygiene of medical interns: Can the sleep, alertness, and fatigue education in residency program help? Arch Intern Med 2007;167:1738-44.

12. Arora V, Dunphy C, Chang VY, et al. The effects of on-duty napping on intern sleep time and fatigue. Ann Intern Med 2006;144:792-8.

13. Richardson GS, Wyatt JK, Sullivan JP, et al. Objective assessment of sleep and alertness in medical house staff and the impact of protected time for sleep. Sleep 1996;19:718-26.

14. Taylor TS, Nisker J, Lingard L. To stay or not to stay? A grounded theory study of residents' postcall behaviors and their rationalizations for those behaviors. Acad Med 2013;88:1529-33.

15. Woodrow SI, Park J, Murray BJ, et al. Differences in the perceived impact of sleep deprivation among surgical and non-surgical residents. Med Educ 2008;42:459-67.

16. Dawson D, Chapman J, Thomas MJ. Fatigue-proofing: a new approach to reducing fatigue-related risk using the principles of error management. Sleep Med Rev 2012;16:167-75.

17. Coverdill JE, Bittner JG, Park MA, et al. Fatigue as impairment or educational necessity? Insights into surgical culture. Acad Med 2011;86(Suppl):S69-72.

18. Charmaz K. Constructing grounded theory: a practical guide through qualitative analysis. Los Angeles: Sage Publications; 2006.

19. Morse JM. The significance of saturation. Qual Health Res 1995;5:147-9.

20. Thurmond VA. The point of triangulation. 7 Nurs Scholarsh 2001;33:253-8.

21. Corbin Dwyer S, Buckle JL. The space between: on being an insider-outsider in qualitative research. Int 7 Qual Methods 2009;8:54-63.

22. Caldwell JA, Caldwell JL. Fatigue in aviation: a guide to staying awake at the stick. Burlington (VT): Ashgate Publishing Company; 2003.

23. Watt CG. Aircrew fatigue management. Montgomery (AL): Air War College, Maxwell Airforce Base; 2009.

24. Osborne R, Parshuram CS. Delinking resident duty hours from patient safety. BMC Med Educ 2014;14(Suppl 1):S2
25. Dawson D. Fatigue research in 2011: from the bench to practice. Accid Anal Prev 2012;45(Suppl):1-5.

26. Teunissen PW. Experience, trajectories, and reifications: an emerging framework of practice-based learning in healthcare workplaces. Adv Health Sci Educ Theory Pract 2015;20:843-56.

Affiliations: Department of Obstetrics \& Gynaecology, and Centre for Education Research \& Innovation (Taylor); Departments of Clinical Neurological Sciences and Oncology (Watling), and Centre for Education Research \& Innovation, Schulich School of Medicine \& Dentistry, Western University, London, Ont.; School of Health Professions Education (Teunissen), Maastricht University, Maastricht; Department of Obstetrics \& Gynaecology (Teunissen), VU University Medical Center, Amsterdam, the Netherlands; Centre for Medical Education (Dornan), Queen's University Belfast, Belfast, Northern Ireland; School of Health Professions Education (Dornan), Maastricht University, Maastricht, the Netherlands; Centre for Education Research \& Innovation (Lingard), Schulich School of Medicine \& Dentistry; Faculty of Education (Lingard), Western University, London, Ont.

Contributors: All of the authors made a substantial contribution to the conception of the study or the data analysis, and to drafting or revising the article. All of the authors gave final approval of the version to be published and agreed to act as guarantors of the work.

Funding: Lorelei Lingard's funding as a scientist in the Program of Experimental Medicine (POEM), from the Department of Medicine at the Schulich School of Medicine \& Dentistry, provided support for this research. A Faculty Support for Research in Education grant from the Schulich School of Medicine \& Dentistry also provided support for this research.

Supplemental information: For reviewer comments and the original submission of this manuscript, please see www.cmajopen.ca/content/4/2/ E200/suppl/DC1. 